\title{
Positivity and Monotonicity Preserving Biquartic Rational Interpolation Spline Surface
}

\author{
Xinru Liu, ${ }^{1,2,3}$ Yuanpeng $\mathrm{Zhu}^{2}$ and Shengjun Liu ${ }^{2,3}$ \\ ${ }^{1}$ Power Metallurgy Research Institute, Central South University, Changsha, Hunan 410083, China \\ ${ }^{2}$ School of Mathematics and Statistics, Central South University, Changsha, Hunan 410083, China \\ ${ }^{3}$ State Key Laboratory of High Performance Complex Manufacturing, Central South University, Changsha, Hunan 410083, China
}

Correspondence should be addressed to Shengjun Liu; shjliu.cg@csu.edu.cn

Received 23 November 2013; Revised 24 February 2014; Accepted 10 March 2014; Published 8 May 2014

Academic Editor: Nazim I. Mahmudov

Copyright (C) 2014 Xinru Liu et al. This is an open access article distributed under the Creative Commons Attribution License, which permits unrestricted use, distribution, and reproduction in any medium, provided the original work is properly cited.

\begin{abstract}
A biquartic rational interpolation spline surface over rectangular domain is constructed in this paper, which includes the classical bicubic Coons surface as a special case. Sufficient conditions for generating shape preserving interpolation splines for positive or monotonic surface data are deduced. The given numeric experiments show our method can deal with surface construction from positive or monotonic data effectively.
\end{abstract}

\section{Introduction}

In most of computer graphics applications and scientific visualization, the construction of shape preserving interpolation spline for positive or monotonic surface data is the essential problem. This problem has been considered by many authors. In [1], the authors gave an algorithm for determining a $C^{1}$ monotone quadratic spline surface interpolating monotone data over a rectangular grid. In [2], Costantini and Fontanella proposed a method for constructing shape preserving surfaces interpolating arbitrary sets of data on rectangular grids. The surfaces are tensor product splines of arbitrary continuity class. In [3], based on the Boolean sum of cubic interpolating operators, Costantini proposed a local method for the construction of differentiable functions which interpolate a set of gridded data and are monotonicity preserving. In [4], Han and Schumaker derived sufficient conditions on the Bézier net of a Bernstein-Bézier polynomial defined on a triangle in the plane to insure that the corresponding surface is monotone. Then, they applied these conditions to construct a new algorithm for fitting a monotone surface to gridded data. In [5], Hussain and Sarfraz gave simple constraints on the free parameters in the description of rational bicubic spline to preserve the shape of positive surface data and to preserve the shape of the data that lie above a plane. A rational bicubic partially blended patch (Coons patch) to visualize the monotone data in the view of monotone surfaces was developed in [6]. The scheme is economical to compute and visually pleasant. In [7], the authors developed a scheme for visualizing positive data set by using a kind of rational cubic trigonometric function. Recently, in [8], Peng et al. developed a $C^{1}$ nonnegativity preserving interpolation spline for nonnegative surface data by using a kind of bivariate rational functions.

The purpose of this paper is to present a kind of quartic rational shape preserving interpolation spline for positive and monotone surface data. It has low computation cost and can generate satisfying shape preserving interpolation spline surface. The rest of this paper is organized as follows. We will construct a class of quartic rational interpolation spline and its error bounds in Section 2. Based on it, Section 3 constructs a biquartic rational interpolation spline over a rectangular domain. After that, the sufficient conditions for constructing shape preserving interpolation splines for positive and monotone surface data are deduced in Sections 4 and 5. The numeric experiments given in Section 6 demonstrate that the biquartic rational interpolation splines are effective for visualizing the positive data and monotone data. Conclusions are given in Section 7. 


\section{Quartic Rational Interpolation Spline and Its Error Bounds}

Let $f_{i} \in \mathbb{R}, i=0,1, \ldots, n$, be data given at the distinct knots $x_{i} \in \mathbb{R}, i=0,1, \ldots, n$, with interval spacing $h_{i}=x_{i+1}-x_{i}>0$, and let $d_{i}$ denote the first derivative values at the knots. The proposed quartic rational interpolation spline is as follows:

$$
\begin{aligned}
H_{i}(x)= & R_{0}\left(t ; \alpha_{i}, \alpha_{i+1}\right) f_{i}+R_{1}\left(t ; \alpha_{i}, \alpha_{i+1}\right)\left(f_{i}+\frac{h_{i}}{2+\alpha_{i}} d_{i}\right) \\
& +R_{2}\left(t ; \alpha_{i}, \alpha_{i+1}\right)\left(f_{i+1}-\frac{h_{i}}{2+\alpha_{i+1}} d_{i+1}\right) \\
& +R_{3}\left(t ; \alpha_{i}, \alpha_{i+1}\right) f_{i+1},
\end{aligned}
$$

where $x \in\left[x_{i}, x_{i+1}\right], t=\left(x-x_{i}\right) / h_{i}, \alpha_{i}, \alpha_{i+1} \in[0,+\infty)$, $i=1,2, \ldots, n-1$, and the quartic rational basis functions $R_{j}\left(t ; \alpha_{j}, \alpha_{j+1}\right)(j=0,1,2,3)$ are given by

$$
\begin{aligned}
& R_{0}\left(t ; \alpha_{i}, \alpha_{i+1}\right)=\frac{(1-t)^{2}}{1+\alpha_{i} t} \\
& R_{1}\left(t ; \alpha_{i}, \alpha_{i+1}\right)=\frac{(1-t)^{2} t}{1+\alpha_{i} t}\left(2+\alpha_{i}+2 \alpha_{i} t\right), \\
& R_{2}\left(t ; \alpha_{i}, \alpha_{i+1}\right)=\frac{(1-t) t^{2}}{1+\alpha_{i+1}(1-t)}\left[2+\alpha_{i+1}+2 \alpha_{i+1}(1-t)\right], \\
& R_{3}\left(t ; \alpha_{i}, \alpha_{i+1}\right)=\frac{t^{2}}{1+\alpha_{i+1}(1-t)} .
\end{aligned}
$$

The spline given in (1) is a $C^{1}$ Hermite interpolation spline as it satisfies the following interpolatory properties:

$$
\begin{array}{ll}
H_{i}\left(x_{i}\right)=f_{i}, & H_{i}\left(x_{i+1}\right)=f_{i+1}, \\
H_{i}^{\prime}\left(x_{i}\right)=d_{i}, & H_{i}^{\prime}\left(x_{i+1}\right)=d_{i+1} .
\end{array}
$$

And it can be easily checked that, for $\alpha_{i}=\alpha_{i+1}=0$, the quartic rational interpolation spline is exactly the classical cubic Hermite interpolation spline.

Now let us assume that the data $\left(x_{i}, f_{i}\right), i=0,1, \ldots, n$, being interpolated are generated from a function $f(x) \in$ $C^{3}\left[x_{0}, x_{n}\right]$. Since the developed interpolation (1) is local, without loss of generality, we only consider the error of approximation in the subinterval $\left[x_{i}, x_{i+1}\right]$. The absolute interpolation error in the subinterval can be expressed in terms of Peano-Kernel [9] as follows:

$$
\left|f(x)-H_{i}(x)\right| \leq \frac{1}{2}\left\|f^{(3)}(\tau)\right\| \int_{x_{i}}^{x_{i+1}}\left|R_{x}\left[(x-\tau)_{+}^{2}\right]\right| d \tau
$$

where $R_{x}\left[(x-\tau)_{+}^{2}\right]$ is known as Peano-Kernel and $(x-\tau)_{+}^{2}$ is the truncated power function. We have $R_{x}\left[(x-\tau)_{+}^{2}\right]=r(\tau, x)$ for $x_{i}<\tau<x$ and $R_{x}\left[(x-\tau)_{+}^{2}\right]=s(\tau, x)$ for $x<\tau<x_{i+1}$. Therefore, the integral involved in (4) can be expressed as

$$
\int_{x_{i}}^{x_{i+1}}\left|R_{x}\left[(x-\tau)_{+}^{2}\right]\right| d \tau=\int_{x_{i}}^{x}|r(\tau, x)| d \tau+\int_{x}^{x_{i+1}}|s(\tau, x)| d \tau .
$$

For the proposed quartic rational interpolation spline (1), we have

$$
\begin{aligned}
& r(\tau, x) \\
& \quad=(x-\tau)^{2} \\
& \quad-\left\{\left[R_{2}(t)+R_{3}(t)\right]\left(x_{i+1}-\tau\right)^{2}-\frac{2 h_{i} R_{2}(t)\left(x_{i+1}-\tau\right)}{2+\alpha_{i+1}}\right\}, \\
& s(\tau, x) \\
& \quad=-\left\{\left[R_{2}(t)+R_{3}(t)\right]\left(x_{i+1}-\tau\right)^{2}-\frac{2 h_{i} R_{2}(t)\left(x_{i+1}-\tau\right)}{2+\alpha_{i+1}}\right\},
\end{aligned}
$$

where $R_{2}(t)$ and $R_{3}(t)$ denote the quartic rational basis functions $R_{2}\left(t ; \alpha_{i}, \alpha_{i+1}\right)$ and $R_{3}\left(t ; \alpha_{i}, \alpha_{i+1}\right)$ given in (2), respectively.

In order to compute the integral of absolute values in (5), the roots of $r(\tau, x)$ and $s(\tau, x)$ are calculated. It is observed that, for all $t \in[0,1], r\left(x, x_{i}\right)=0$. Substituting $\tau=x$ in (6) and after some simplification, we have

$$
r(x, x)=-h_{i}^{2}(1-t)\left\{\left[R_{2}(t)+R_{3}(t)\right](1-t)-\frac{2 R_{2}(t)}{2+\alpha_{i+1}}\right\} .
$$

Therefore, the roots of $r(x, x)$ in $[0,1]$ are $t=0, t=1$, and

$$
t^{*}=1-\frac{-\left(4+\alpha_{i+1}^{2}\right)+\sqrt{\left(4+\alpha_{i+1}^{2}\right)^{2}+8 \alpha_{i+1}\left(2+\alpha_{i+1}\right)^{2}}}{4 \alpha_{i+1}\left(2+\alpha_{i+1}\right)} .
$$

Similarly, we have $s\left(x, x_{i+1}\right)=0$. And it is obvious that the roots of $s(x, x)$ in $[0,1]$ are also $t=0, t=1$, and $t^{*}$. To compute the roots of $r(\tau, x)$, we rewrite it as the following form:

$$
\begin{aligned}
r(\tau, x)= & {\left[1-R_{2}(t)-R_{3}(t)\right](x-\tau)^{2} } \\
& +2 h_{i}\left\{\frac{R_{2}(t)}{2+\alpha_{i+1}}-\left[R_{2}(t)+R_{3}(t)\right](1-t)\right\}(x-\tau) \\
& +h_{i}^{2}\left\{\frac{2 R_{2}}{2+\alpha_{i+1}}(1-t)-\left[R_{2}(t)+R_{3}(t)\right](1-t)^{2}\right\} .
\end{aligned}
$$

Thus, we can get the roots of $r(\tau, x)$ as follows:

$$
\tau_{1}^{*}=x+h_{i}\left(\frac{B-D}{A}\right), \quad \tau_{2}^{*}=x+h_{i}\left(\frac{B+D}{A}\right),
$$

where

$$
\begin{aligned}
A= & -R_{2}(t)-R_{3}(t), \\
B= & \frac{R_{2}(t)}{2+\alpha_{i+1}}-\left[R_{2}(t)+R_{3}(t)\right](1-t), \\
D=( & {\left[\frac{R_{2}}{2+\alpha_{i+1}}-\left(R_{2}+R_{3}\right)(1-t)\right]^{2} } \\
& \quad\left(1-R_{2}-R_{3}\right) \\
& \left.\times\left[\frac{2 R_{2}}{2+\alpha_{i+1}}-\left(R_{2}+R_{3}\right)(1-t)\right]\right)^{1 / 2} .
\end{aligned}
$$


Moreover, we can easily obtain the following roots of $s(\tau, x)$ :

$$
\tau=x_{i+1}, \quad \tau^{*}=x_{i+1}-\frac{2 h_{i} R_{2}(t)}{\left(2+\alpha_{i+1}\right)\left[R_{2}(t)+R_{3}(t)\right]} .
$$

The above discussion provides the different values of the absolute error as follows.

Case 1. For $0 \leq t \leq t^{*}$, the absolute error (4) in $\left[x_{i}, x_{i+1}\right]$ is

$$
\left|f(x)-H_{i}(x)\right| \leq \frac{1}{2}\left\|f^{(3)}(t)\right\| h_{i}^{3} \omega_{1}\left(\alpha_{i}, \alpha_{i+1}, t\right),
$$

where

$$
\begin{aligned}
& \omega_{1}\left(\alpha_{i}, \alpha_{i+1}, t\right)=\int_{x_{i}}^{x}|r(\tau, x)| d \tau+\int_{x}^{x_{i+1}}|s(\tau, x)| d \tau \\
& =-\int_{x_{i}}^{\tau_{1}^{*}} r(\tau, x) d \tau+\int_{\tau_{1}^{*}}^{x} r(\tau, x) d \tau \\
& -\int_{x}^{\tau^{*}} s(\tau, x) d \tau+\int_{\tau^{*}}^{x_{i+1}} s(\tau, x) d \tau \\
& =-\left(\frac{1-R_{2}-R_{3}}{3}\right) t^{3}+\left(\frac{R_{2}+R_{3}}{3}\right)(1-t)^{3} \\
& -\left[\frac{R_{2}}{2+\alpha_{i+1}}-\left(R_{2}+R_{3}\right)(1-t)\right] t^{2} \\
& -\frac{R_{2}}{2+\alpha_{i+1}}(1-t)^{2} \\
& -\left[\frac{2 R_{2}(1-t)}{2+\alpha_{i+1}}-\left(R_{2}+R_{3}\right)(1-t)^{2}\right] t \\
& +\frac{8 R_{2}^{3}}{3\left(2+\alpha_{i+1}\right)^{3}\left(R_{2}+R_{3}\right)^{2}} \\
& -\frac{2\left(1-R_{2}-R_{3}\right)}{3}\left(\frac{B-D}{A}\right)^{3} \\
& +2\left[\frac{R_{2}}{2+\alpha_{i+1}}-\left(R_{2}+R_{3}\right)(1-t)\right] \\
& \times\left(\frac{B-D}{A}\right)^{2} \\
& -2\left[\frac{2 R_{2}(1-t)}{2+\alpha_{i+1}}-\left(R_{2}+R_{3}\right)(1-t)^{2}\right] \\
& \times\left(\frac{B-D}{A}\right) .
\end{aligned}
$$

Case 2. For $t^{*} \leq t \leq 1$, the absolute error (4) in $\left[x_{i}, x_{i+1}\right]$ is

$$
\left|f(x)-H_{i}(x)\right| \leq \frac{1}{2}\left\|f^{(3)}(t)\right\| h_{i}^{3} \omega_{2}\left(\alpha_{i}, \alpha_{i+1}, t\right),
$$

where

$$
\begin{aligned}
& \omega_{2}\left(\alpha_{i}, \alpha_{i+1}, t\right)=\int_{x_{i}}^{x}|r(\tau, x)| d \tau+\int_{x}^{x_{i+1}}|s(\tau, x)| d \tau \\
& =\int_{x_{i}}^{\tau_{1}^{*}} r(\tau, x) d \tau-\int_{\tau_{1}^{*}}^{\tau_{2}^{*}} r(\tau, x) d \tau \\
& +\int_{\tau_{2}^{*}}^{x} s(\tau, x) d \tau+\int_{x}^{x_{i+1}} s(\tau, x) d \tau \\
& =\left(\frac{1-R_{2}-R_{3}}{3}\right) t^{3} \\
& +\left[\frac{R_{2}}{2+\alpha_{i+1}}-\left(R_{2}+R_{3}\right)(1-t)\right] t^{2} \\
& +\left[\frac{3 R_{2}-\left(2+\alpha_{i+1}\right)\left(R_{2}+R_{3}\right)(1-t)}{3\left(2+\alpha_{i+1}\right)}\right] \\
& \times(1-t)^{2} \\
& +\left[\frac{2 R_{2}(1-t)}{2+\alpha_{i+1}}-\left(R_{2}+R_{3}\right)(1-t)^{2}\right] t \\
& +\frac{2\left(1-R_{2}-R_{3}\right)}{3}\left(\frac{B-D}{A}\right)^{3} \\
& -2\left[\frac{R_{2}}{2+\alpha_{i+1}}-\left(R_{2}+R_{3}\right)(1-t)\right] \\
& \times\left(\frac{B-D}{A}\right)^{2} \\
& +\left[\frac{2 R_{2}(1-t)}{2+\alpha_{i+1}}-\left(R_{2}+R_{3}\right)(1-t)^{2}\right] \\
& \times\left(\frac{B-D}{A}\right) \\
& -\frac{2\left(1-R_{2}-R_{3}\right)}{3}\left(\frac{B+D}{A}\right)^{3} \\
& +2\left[\frac{R_{2}}{2+\alpha_{i+1}}-\left(R_{2}+R_{3}\right)(1-t)\right] \\
& \times\left(\frac{B+D}{A}\right)^{2} \\
& -\left[\frac{2 R_{2}(1-t)}{2+\alpha_{i+1}}-\left(R_{2}+R_{3}\right)(1-t)^{2}\right] \\
& \times\left(\frac{B+D}{A}\right) \text {. }
\end{aligned}
$$

Summarizing the above discussion, we conclude the following theorem.

Theorem 1. For $f(x) \in C^{3}\left[x_{0}, x_{n}\right]$, let $H_{i}(x)$ be the quartic rational interpolating function of $f(t)$ in $\left[x_{i}, x_{i+1}\right]$ defined by (1). For the nonnegative parameter $\alpha_{i}$, the error of the interpolating function $H_{i}(x)$ satisfies

$$
\begin{gathered}
\left|f(x)-H_{i}(x)\right| \leq \frac{1}{2}\left\|f^{(3)}(t)\right\| h_{i}^{3} c_{i}, \\
c_{i}=\max _{0 \leq t \leq 1} \omega\left(\alpha_{i}, \alpha_{i+1}, t\right),
\end{gathered}
$$

with

$$
\omega\left(\alpha_{i}, \alpha_{i+1}, t\right)= \begin{cases}\omega_{1}\left(\alpha_{i}, \alpha_{i+1}, t\right), & 0 \leq t \leq t^{*} \\ \omega_{2}\left(\alpha_{i}, \alpha_{i+1}, t\right), & t^{*} \leq t \leq 1\end{cases}
$$




\section{Biquartic Rational Interpolation Spline}

Let $\left\{\left(x_{i}, y_{i}, F_{i j}\right), i=0,1,2, \ldots, m ; j=0,1,2, \ldots, n\right\}$ be a given set of data points defined over the rectangular domain $D=$ $[a, b] \times[c, d]$, where $a=x_{0}<x_{1}<x_{2}<\cdots<x_{m}=b$ and $c=y_{0}<y_{1}<y_{2}<\cdots<y_{n}=d$. The biquartic rational interpolation spline $R(x, y)$ is defined over each rectangular patch $I_{i j}=\left[x_{i}, x_{i+1}\right] \times\left[y_{j}, y_{j+1}\right](i=0,1,2, \ldots, m-1 ; j=$ $0,1,2, \ldots, n-1)$ as

$$
R(x, y)=R_{i, j}(x, y)=I_{i, j}(u) F(i, j) \widehat{I}_{i, j}^{T}(v),
$$

where

$$
\begin{aligned}
& u=\frac{x-x_{i}}{h_{i}}, \quad v=\frac{y-y_{j}}{\widehat{h}_{j}}, \quad h_{i}=x_{i+1}-x_{i}, \\
& \widehat{h}_{j}=y_{j+1}-y_{j}, \\
& F(i, j)=\left[\begin{array}{cccc}
F_{i, j} & F_{i, j+1} & F_{i, j}^{y} & F_{i, j+1}^{y} \\
F_{i+1, j} & F_{i+1, j+1} & F_{i+1, j}^{y} & F_{i+1, j+1}^{y} \\
F_{i, j}^{x} & F_{i, j+1}^{x} & F_{i, j}^{x y} & F_{i, j+1}^{x y} \\
F_{i+1, j}^{x} & F_{i+1, j+1}^{x} & F_{i+1, j}^{x y} & F_{i+1, j+1}^{x y}
\end{array}\right] \text {, } \\
& I_{i, j}(u)=\left[\begin{array}{llll}
I_{i 0}(u) & I_{i 1}(u) & I_{i 2}\left(u ; \alpha_{i, j}\right) & I_{i 3}\left(u ; \beta_{i, j}\right)
\end{array}\right] \text {, } \\
& \widehat{I}_{i, j}(v)=\left[\widehat{I}_{0 j}(v) \widehat{I}_{1 j}(v) \widehat{I}_{2 j}\left(v ; \widehat{\alpha}_{i, j}\right) \widehat{I}_{3 j}\left(v ; \widehat{\beta}_{i, j}\right)\right] \text {, }
\end{aligned}
$$

with

$$
\begin{aligned}
I_{i 0}(u) & =1-3 u^{2}+2 u^{3}, \\
I_{i 1}(u) & =3 u^{2}-2 u^{3} \\
I_{i 2}\left(u ; \alpha_{i, j}\right) & =\frac{h_{i}(1-u)^{2} u\left(2+\alpha_{i, j}+2 \alpha_{i, j} u\right)}{\left(2+\alpha_{i, j}\right)\left(1+\alpha_{i, j} u\right)}, \\
I_{i 3}\left(u ; \beta_{i, j}\right) & =\frac{-h_{i}(1-u) u^{2}\left[2+\beta_{i, j}+2 \beta_{i, j}(1-u)\right]}{\left(2+\beta_{i, j}\right)\left[1+\beta_{i, j}(1-u)\right]}, \\
\widehat{I}_{0 j}(v) & =1-3 v^{2}+2 v^{3}, \\
\widehat{I}_{1 j}(v) & =3 v^{2}-2 v^{3}, \\
\widehat{I}_{2 j}\left(v ; \widehat{\alpha}_{i, j}\right) & =\frac{\widehat{h}_{j}(1-v)^{2} v\left(2+\widehat{\alpha}_{i, j}+2 \widehat{\alpha}_{i, j} v\right)}{\left(2+\widehat{\alpha}_{i, j}\right)\left(1+\widehat{\alpha}_{i, j} v\right)} \\
\widehat{I}_{3 j}\left(v ; \widehat{\beta}_{i, j}\right) & =\frac{-\widehat{h}_{j}(1-v) v^{2}\left[2+\widehat{\beta}_{i, j}+2 \widehat{\beta}_{i, j}(1-v)\right]}{\left(2+\widehat{\beta}_{i, j}\right)\left[1+\beta_{i, j}(1-v)\right]} .
\end{aligned}
$$

Here, $F_{i, j}$ are known as the given data, $F_{i, j}^{x}, F_{i, j}^{y}$ are the first derivatives, and $F_{i, j}^{x y}$ are the mixed derivatives. Obviously, for all $\alpha_{i, j}=\widehat{\alpha}_{i, j}=\beta_{i, j}=\widehat{\beta}_{i, j}=0$, the new proposed patch $R_{i, j}(x, y)$ is exactly the classical bicubic Coons patch.

In most applications, the derivative parameters $F_{i, j}^{x}, F_{i, j}^{y}$, and $F_{i, j}^{x y}$ are not given and hence must be determined either from given data or by some other means. These methods are the approximation based on various mathematical theories. We use a common choice as follows:

$$
\begin{aligned}
F_{0, j}^{x}= & \Delta_{0, j}+\left(\Delta_{0, j}-\Delta_{1, j}\right) \frac{h_{0}}{\left(h_{0}+h_{1}\right)}, \\
F_{m, j}^{x}= & \Delta_{m-1, j}+\left(\Delta_{m-1, j}-\Delta_{m-2, j}\right) \frac{h_{m-1}}{\left(h_{m-1}+h_{m-2}\right)}, \\
F_{i, j}^{x}= & \frac{\Delta_{i, j}+\Delta_{i-1, j}}{2}, \quad i=1,2,3, \ldots, m-1 ; j=0,1,2, \ldots, n, \\
F_{i, 0}^{y}= & \widehat{\Delta}_{i, 0}+\left(\widehat{\Delta}_{i, 0}-\widehat{\Delta}_{i, 1}\right) \frac{\widehat{h}_{0}}{\left(\widehat{h}_{0}+\widehat{h}_{1}\right)}, \\
F_{i, n}^{y}= & \widehat{\Delta}_{i, n-1}+\left(\widehat{\Delta}_{i, n-1}-\widehat{\Delta}_{i, n-2}\right) \frac{\widehat{h}_{n-1}}{\left(\widehat{h}_{n-1}+\widehat{h}_{n-2}\right)}, \\
F_{i, j}^{y}= & \frac{\widehat{\Delta}_{i, j}+\widehat{\Delta}_{i, j-1}, \quad i=0,1,2, \ldots, m ; j=1,2,3, \ldots, n-1,}{2} \\
& i=1,2,3, \ldots, m-1 ; j=1,2,3, \ldots, n-1, \\
& F_{i, j}^{x y}=\frac{1}{2}\left[\frac{F_{i, j+1}^{x}-F_{i, j-1}^{x}}{\widehat{h}_{j-1}+\widehat{h}_{j}}+\frac{F_{i+1, j}^{y}-F_{i-1, j}^{y}}{h_{i-1}+h_{i}}\right],
\end{aligned}
$$

where $\Delta_{i, j}=\left(F_{i+1, j}-F_{i, j}\right) / h_{i}$ and $\widehat{\Delta}_{i, j}=\left(F_{i, j+1}-F_{i, j}\right) / \widehat{h}_{j}$. These arithmetic mean methods are computationally economical and suitable for visualization of shaped data.

For convenience, by using the quartic rational basis functions (2), we rewrite the quartic rational interpolation spline $R(x, y)$ given in (20) as follows:

$$
\begin{aligned}
R(x, y)= & R_{0}\left(u ; \alpha_{i, j}, \beta_{i, j}\right) \phi_{i, j}+R_{1}\left(u ; \alpha_{i, j}, \beta_{i, j}\right) \varphi_{i, j} \\
& +R_{2}\left(u ; \alpha_{i, j}, \beta_{i, j}\right) \xi_{i, j}+R_{3}\left(u ; \alpha_{i, j}, \beta_{i, j}\right) \zeta_{i, j},
\end{aligned}
$$

where

$$
\phi_{i, j}=\sum_{k=0}^{3} R_{k}\left(v ; \widehat{\alpha}_{i, j} ; \widehat{\beta}_{i, j}\right) A_{0, k}
$$

with

$$
\begin{aligned}
& A_{0,0}=F_{i, j}, \\
& A_{0,1}=F_{i, j}+\frac{\widehat{h}_{j}}{2+\widehat{\alpha}_{i, j}} F_{i, j}^{y}, \\
& A_{0,2}=F_{i, j+1}-\frac{\widehat{h}_{j}}{2+\widehat{\beta}_{i, j}} F_{i, j+1}^{y}, \\
& A_{0,3}=F_{i, j+1}, \\
& \varphi_{i, j}=\sum_{k=0}^{3} R_{k}\left(v ; \widehat{\alpha}_{i, j} ; \widehat{\beta}_{i, j}\right) A_{1, k},
\end{aligned}
$$


with

$$
\begin{aligned}
A_{1,0}= & F_{i, j}+\frac{h_{i}}{2+\alpha_{i, j}} F_{i, j}^{x}, \\
A_{1,1}= & \left(F_{i, j}+\frac{h_{i}}{2+\alpha_{i, j}} F_{i, j}^{x}\right)+\frac{\widehat{h}_{j}}{2+\widehat{\alpha}_{i, j}}\left(F_{i, j}^{y}+\frac{h_{i}}{2+\alpha_{i, j}} F_{i, j}^{x y}\right), \\
A_{1,2}= & \left(F_{i, j+1}+\frac{h_{i}}{2+\alpha_{i, j}} F_{i, j+1}^{x}\right) \\
& -\frac{\widehat{h}_{j}}{2+\widehat{\beta}_{i, j}}\left(F_{i, j+1}^{y}+\frac{h_{i}}{2+\alpha_{i, j}} F_{i, j+1}^{x y}\right), \\
A_{1,3}= & F_{i, j+1}+\frac{h_{i}}{2+\alpha_{i, j}} F_{i, j+1}^{x}, \\
\xi_{i, j}= & \sum_{k=0}^{3} R_{k}\left(v ; \widehat{\alpha}_{i, j} ; \widehat{\beta}_{i, j}\right) A_{2, k},
\end{aligned}
$$

with

$$
\begin{aligned}
A_{2,0}= & F_{i+1, j}-\frac{h_{i}}{2+\beta_{i, j}} F_{i+1, j}^{x}, \\
A_{2,1}= & \left(F_{i+1, j}-\frac{h_{i}}{2+\beta_{i, j}} F_{i+1, j}^{x}\right) \\
& +\frac{\widehat{h}_{j}}{2+\widehat{\alpha}_{i, j}}\left(F_{i+1, j}^{y}-\frac{h_{i}}{2+\beta_{i, j}} F_{i+1, j}^{x y}\right), \\
A_{2,2}= & \left(F_{i+1, j+1}-\frac{h_{i}}{2+\beta_{i, j}} F_{i+1, j+1}^{x}\right) \\
& -\frac{\widehat{h}_{j}}{2+\widehat{\beta}_{i, j}}\left(F_{i+1, j+1}^{y}-\frac{h_{i}}{2+\beta_{i, j}} F_{i+1, j+1}^{x y}\right), \\
A_{2,3}= & F_{i+1, j+1}-\frac{h_{i}}{2+\beta_{i, j}} F_{i+1, j+1}^{x}, \\
\zeta_{i, j}= & \sum_{k=0}^{3} R_{k}\left(v ; \widehat{\alpha}_{i, j} ; \widehat{\beta}_{i, j}\right) A_{3, k},
\end{aligned}
$$

with

$$
\begin{aligned}
& A_{3,0}=F_{i+1, j}, \\
& A_{3,1}=F_{i+1, j}+\frac{\widehat{h}_{j}}{2+\widehat{\alpha}_{i, j}} F_{i+1, j}^{y}, \\
& A_{3,2}=F_{i+1, j+1}-\frac{\widehat{h}_{j}}{2+\widehat{\beta}_{i, j}} F_{i+1, j+1}^{y}, \\
& A_{3,3}=F_{i+1, j+1} .
\end{aligned}
$$

\section{Positivity Preserving Interpolation}

Let $\left\{\left(x_{i}, y_{i}, F_{i, j}\right), i=0,1,2, \ldots, m ; j=0,1,2, \ldots, n\right\}$ be positive data defined over the rectangular domain $D=$ $\left[x_{0}, x_{m}\right] \times\left[y_{0}, y_{n}\right]$ such that

$$
F_{i, j}>0, \quad \forall i, j .
$$

The quartic rational interpolation spline (24) preserves the shape of positive data if

$$
R(x, y)>0, \quad \forall(x, y) \in D .
$$

For any $\alpha, \beta \in[0,+\infty)$, since the quartic rational basis functions given in (2) have the property of nonnegativity, from (24) we can see that a sufficient condition for $R(x, y)>0$ is

$$
A_{l, k}>0, \quad l, k=0,1,2,3 .
$$

For $A_{0, k}>0, k=0,1,2,3$, a sufficient condition is

$$
\begin{aligned}
& \widehat{\alpha}_{i, j}>\max \left\{0,-2-\frac{\widehat{h}_{j} F_{i, j}^{y}}{F_{i, j}}\right\}, \\
& \widehat{\beta}_{i, j}>\max \left\{0,-2+\frac{\widehat{h}_{j} F_{i, j+1}^{y}}{F_{i, j+1}}\right\} .
\end{aligned}
$$

For $A_{1, k}>0, k=0,1,2,3$, a sufficient condition is

$$
\begin{aligned}
& \alpha_{i, j}>\max \left\{0,-2-\frac{h_{i} F_{i, j}^{x}}{F_{i, j}},-2-\frac{h_{i} F_{i, j+1}^{x}}{F_{i, j+1}}\right\}, \\
& \widehat{\alpha}_{i, j}>\max \left\{0,-2-\frac{\widehat{h}_{j}\left[\left(2+\alpha_{i, j}\right) F_{i, j}^{y}+h_{i} F_{i, j}^{x y}\right]}{\left(2+\alpha_{i, j}\right) F_{i, j}+h_{i} F_{i, j}^{x}}\right\}, \\
& \widehat{\beta}_{i, j}>\max \left\{0,-2+\frac{\widehat{h}_{j}\left[\left(2+\alpha_{i, j}\right) F_{i, j+1}^{y}+h_{i} F_{i, j+1}^{x y}\right]}{\left(2+\alpha_{i, j}\right) F_{i, j+1}+h_{i} F_{i, j+1}^{x}}\right\} .
\end{aligned}
$$

For $A_{2, k}>0, k=0,1,2,3$, a sufficient condition is

$$
\begin{aligned}
& \beta_{i, j}>\max \left\{0,-2+\frac{h_{i} F_{i+1, j}^{x}}{F_{i+1, j}},-2+\frac{h_{i} F_{i+1, j+1}^{x}}{F_{i+1, j+1}}\right\}, \\
& \widehat{\alpha}_{i, j}>\max \left\{0, \frac{\widehat{h}_{j}\left[\left(2+\beta_{i, j}\right) F_{i+1, j}^{y}-h_{i} F_{i+1, j}^{x y}\right]}{\left(2+\beta_{i, j}\right) F_{i+1, j}-h_{i} F_{i+1, j}^{x}}\right\}, \\
& \widehat{\beta}_{i, j}>\max \left\{0, \frac{\widehat{h}_{j}\left[\left(2+\beta_{i, j}\right) F_{i+1, j+1}^{y}-h_{i} F_{i+1, j+1}^{x y}\right]}{\left(2+\beta_{i, j}\right) F_{i+1, j+1}-h_{i} F_{i+1, j+1}^{x}}\right\} .
\end{aligned}
$$

For $A_{3, k}>0, k=0,1,2,3$, a sufficient condition is

$$
\begin{aligned}
& \widehat{\alpha}_{i, j}>\max \left\{0,-2-\frac{\widehat{h}_{j} F_{i+1, j}^{y}}{F_{i+1, j}}\right\}, \\
& \widehat{\beta}_{i, j}>\max \left\{0,-2+\frac{\widehat{h}_{j} F_{i+1, j+1}^{y}}{F_{i+1, j+1}}\right\} .
\end{aligned}
$$

Summarizing the above discussion, we can conclude the following theorem.

Theorem 2. The biquartic rational interpolation spline defined in (24) visualizes positive data in the view of positive surface if in each rectangular patch $I_{i, j}=\left[x_{i}, x_{i+1}\right] \times\left[y_{j}, y_{j+1}\right](i=$ 
$0,1, \ldots, m-1 ; j=0,1, \ldots, n-1)$ the shape parameters $\alpha_{i, j}$, $\beta_{i, j}, \widehat{\alpha}_{i, j}$, and $\widehat{\beta}_{i, j}$ satisfy the following conditions:

$$
\begin{aligned}
& \alpha_{i, j}=m_{i, j}+\max \left\{0,-2-\frac{h_{i} F_{i, j}^{x}}{F_{i, j}},-2-\frac{h_{i} F_{i, j+1}^{x}}{F_{i, j+1}}\right\}, \\
& \beta_{i, j}=n_{i, j}+\max \left\{0,-2+\frac{h_{i} F_{i+1, j}^{x}}{F_{i+1, j}},-2+\frac{h_{i} F_{i+1, j+1}^{x}}{F_{i+1, j+1}}\right\} \text {, } \\
& \widehat{\alpha}_{i, j}=\widehat{m}_{i, j} \\
& +\max \left\{0,-2-\frac{\widehat{h}_{j} F_{i, j}^{y}}{F_{i, j}},\right. \\
& -2-\frac{\widehat{h}_{j}\left[\left(2+\alpha_{i, j}\right) F_{i, j}^{y}+h_{i} F_{i, j}^{x y}\right]}{\left(2+\alpha_{i, j}\right) F_{i, j}+h_{i} F_{i, j}^{x}}, \\
& \frac{\widehat{h}_{j}\left[\left(2+\beta_{i, j}\right) F_{i+1, j}^{y}-h_{i} F_{i+1, j}^{x y}\right]}{\left(2+\beta_{i, j}\right) F_{i+1, j}-h_{i} F_{i+1, j}^{x}} \text {, } \\
& \left.-2-\frac{\widehat{h}_{j} F_{i+1, j}^{y}}{F_{i+1, j}}\right\} \text {, } \\
& \widehat{\beta}_{i, j}=\widehat{n}_{i, j} \\
& +\max \left\{0,-2+\frac{\widehat{h}_{j} F_{i, j+1}^{y}}{F_{i, j+1}},\right. \\
& -2+\frac{\widehat{h}_{j}\left[\left(2+\alpha_{i, j}\right) F_{i, j+1}^{y}+h_{i} F_{i, j+1}^{x y}\right]}{\left(2+\alpha_{i, j}\right) F_{i, j+1}+h_{i} F_{i, j+1}^{x}}, \\
& \frac{\widehat{h}_{j}\left[\left(2+\beta_{i, j}\right) F_{i+1, j+1}^{y}-h_{i} F_{i+1, j+1}^{x y}\right]}{\left(2+\beta_{i, j}\right) F_{i+1, j+1}-h_{i} F_{i+1, j+1}^{x}}, \\
& \left.-2+\frac{\widehat{h}_{j} F_{i+1, j+1}^{y}}{F_{i+1, j+1}}\right\} \text {, }
\end{aligned}
$$

where $m_{i j}, n_{i j}, \widehat{m}_{i j}, \widehat{n}_{i j}>0$ are left to users for interactively controlling the shape of the obtained positivity preserving interpolation spline surface.

\section{Monotonicity Preserving Interpolation}

Let $\left\{\left(x_{i}, y_{i}, F_{i j}\right), i=0,1,2, \ldots, m ; j=0,1,2, \ldots, n\right\}$ be a monotone data defined over the rectangular domain $D=$ $\left[x_{0}, x_{m}\right] \times\left[y_{0}, y_{n}\right]$. The necessary conditions for monotonicity of the data are

$$
\begin{gathered}
F_{i+1, j}>F_{i, j}, \quad \Delta_{i, j}>0, \quad F_{i, j}^{x}>0, \\
F_{i, j+1}>F_{i, j}, \quad \widehat{\Delta}_{i, j}>0, \quad F_{i, j}^{y}>0, \quad \forall i, j .
\end{gathered}
$$

The biquartic rational interpolation spline $R(x, y)$ preserves the shape of monotone surface data if

$$
\begin{array}{r}
\frac{\partial R(x, y)}{\partial x}=R_{x}(x, y)>0, \quad \frac{\partial R(x, y)}{\partial y}= \\
R_{y}(x, y)>0, \\
\forall(x, y) \in D .
\end{array}
$$

Straightforward computation gives that

$$
\begin{aligned}
R_{x}(x, y)= & \frac{(1-u)\left(2+\alpha_{i, j}+\alpha_{i, j} u\right)}{\left(2+\alpha_{i, j}\right)\left(1+\alpha_{i, j} u\right)^{2}} \widehat{\psi}_{i, j} \\
& +\frac{u\left[2+\beta_{i, j}+\beta_{i, j}(1-u)\right]}{\left(2+\beta_{i+1}\right)\left[1+\beta_{i, j}(1-u)\right]^{2}} \widehat{\tau}_{i, j} \\
& +6(1-u) u \widehat{\rho}_{i, j},
\end{aligned}
$$

where

$$
\widehat{\psi}_{i, j}=\sum_{k=0}^{3} R_{k}\left(v ; \widehat{\alpha}_{i, j}, \widehat{\beta}_{i, j}\right) \widehat{C}_{0, k},
$$

with

$$
\begin{gathered}
\widehat{C}_{0,0}=F_{i, j}^{x}, \quad \widehat{C}_{0,1}=F_{i, j}^{x}+\frac{\widehat{h}_{j}}{2+\widehat{\alpha}_{i, j}} F_{i, j}^{x y}, \\
\widehat{C}_{0,2}=F_{i, j+1}^{x}-\frac{\widehat{h}_{j}}{2+\widehat{\beta}_{i, j}} F_{i, j+1}^{x y}, \quad \widehat{C}_{0,3}=F_{i, j+1}^{x}, \\
\widehat{\tau}_{i, j}=\sum_{k=0}^{3} R_{k}\left(v ; \widehat{\alpha}_{i, j}, \widehat{\beta}_{i, j}\right) \widehat{C}_{1, k},
\end{gathered}
$$

with

$$
\begin{gathered}
\widehat{C}_{1,0}=F_{i+1, j}^{x}, \quad \widehat{C}_{1,1}=F_{i+1, j}^{x}+\frac{\widehat{h}_{j}}{2+\widehat{\alpha}_{i, j}} F_{i+1, j}^{x y}, \\
\widehat{C}_{1,2}=F_{i+1, j+1}^{x}-\frac{\widehat{h}_{j}}{2+\widehat{\beta}_{i, j}} F_{i+1, j+1}^{x y}, \quad \widehat{C}_{1,3}=F_{i+1, j+1}^{x}, \\
\widehat{\rho}_{i, j}=\sum_{k=0}^{3} R_{k}\left(v ; \widehat{\alpha}_{i, j}, \widehat{\beta}_{i, j}\right) \widehat{C}_{2, k},
\end{gathered}
$$

with

$$
\begin{gathered}
\widehat{C}_{2,0}=\Delta_{i, j}-\left(\frac{F_{i, j}^{x}}{2+\alpha_{i, j}}+\frac{F_{i+1, j}^{x}}{2+\beta_{i, j}}\right), \\
\widehat{C}_{2,1}=\Delta_{i, j}-\left(\frac{F_{i, j}^{x}}{2+\alpha_{i, j}}+\frac{F_{i+1, j}^{x}}{2+\beta_{i, j}}\right) \\
+\frac{\widehat{h}_{j}}{2+\widehat{\alpha}_{i, j}}\left[\frac{F_{i+1, j}^{y}-F_{i, j}^{y}}{h_{i}}-\left(\frac{F_{i, j}^{x y}}{2+\alpha_{i, j}}+\frac{F_{i+1, j}^{x y}}{2+\beta_{i, j}}\right)\right], \\
\widehat{C}_{2,2}=\Delta_{i, j+1}-\left(\frac{F_{i, j+1}^{x}}{2+\alpha_{i, j}}+\frac{F_{i+1, j+1}^{x}}{2+\beta_{i, j}}\right) \\
-\frac{\widehat{h}_{j}}{2+\widehat{\beta}_{i, j}} \\
\times\left[\frac{\left.F_{i+1, j+1}^{y}-F_{i, j+1}^{y}-\left(\frac{F_{i, j+1}^{x y}}{2+\alpha_{i, j}}+\frac{F_{i+1, j+1}^{x y}}{2+\beta_{i, j}}\right)\right],}{h_{i}}\right. \\
\widehat{C}_{2,3}=\Delta_{i, j+1}-\left(\frac{F_{i, j+1}^{x}}{2+\alpha_{i, j}}+\frac{F_{i+1, j+1}^{x}}{2+\beta_{i, j}}\right) .
\end{gathered}
$$


Similarly, we have

$$
\begin{aligned}
R_{y}(x, y)= & \frac{(1-v)\left(2+\widehat{\alpha}_{i, j}+\widehat{\alpha}_{i, j} v\right)}{\left(2+\widehat{\alpha}_{i, j}\right)\left(1+\widehat{\alpha}_{i, j} v\right)^{2}} \psi_{i, j} \\
& +\frac{v\left[2+\widehat{\beta}_{i, j}+\widehat{\beta}_{i, j}(1-v)\right]}{\left(2+\widehat{\beta}_{i, j}\right)\left[1+\widehat{\beta}_{i, j}(1-v)\right]^{2}} \tau_{i, j} \\
& +6(1-v) v \rho_{i, j},
\end{aligned}
$$

where

$$
\psi_{i, j}=\sum_{k=0}^{3} R_{k}\left(u ; \alpha_{i, j}, \beta_{i, j}\right) C_{0, k}
$$

with

$$
\begin{gathered}
C_{0,0}=F_{i, j}^{y}, \quad C_{0,1}=F_{i, j}^{y}+\frac{h_{i}}{2+\alpha_{i, j}} F_{i, j}^{x y}, \\
C_{0,2}=F_{i+1, j}^{y}-\frac{h_{i}}{2+\beta_{i, j}} F_{i+1, j}^{x y}, \quad C_{0,3}=F_{i+1, j}^{y}, \\
\tau_{i, j}=\sum_{k=0}^{3} R_{k}\left(u ; \alpha_{i, j}, \beta_{i, j}\right) C_{1, k},
\end{gathered}
$$

with

$$
\begin{gathered}
C_{1,0}=F_{i, j+1}^{y}, \quad C_{1,1}=F_{i, j+1}^{y}+\frac{h_{i}}{2+\alpha_{i, j}} F_{i, j+1}^{x y}, \\
C_{1,2}=F_{i+1, j+1}^{y}-\frac{h_{i}}{2+\beta_{i, j}} F_{i+1, j+1}^{x y}, \quad C_{1,3}=F_{i+1, j+1}^{y}, \\
\rho_{i, j}=\sum_{k=0}^{3} R_{k}\left(u ; \alpha_{i, j}, \beta_{i, j}\right) C_{2, k},
\end{gathered}
$$

with

$$
\begin{aligned}
C_{2,0}= & \widehat{\Delta}_{i, j}-\left(\frac{F_{i, j}^{y}}{2+\widehat{\alpha}_{i, j}}+\frac{F_{i, j+1}^{y}}{2+\widehat{\beta}_{i, j}}\right), \\
C_{2,1}= & \widehat{\Delta}_{i, j}-\left(\frac{F_{i, j}^{y}}{2+\widehat{\alpha}_{i, j}}+\frac{F_{i, j+1}^{y}}{2+\widehat{\beta}_{i, j}}\right) \\
& +\frac{h_{i}}{2+\alpha_{i, j}}\left[\frac{F_{i, j+1}^{x}-F_{i, j}^{x}}{\widehat{h}_{j}}-\left(\frac{F_{i, j}^{x y}}{2+\widehat{\alpha}_{i, j}}+\frac{F_{i, j+1}^{x y}}{2+\widehat{\beta}_{i, j}}\right)\right], \\
C_{2,2}= & \widehat{\Delta}_{i+1, j}-\left(\frac{F_{i+1, j}^{y}}{2+\widehat{\alpha}_{i, j}}+\frac{F_{i+1, j+1}^{y}}{2+\widehat{\beta}_{i, j}}\right) \\
& -\frac{h_{i}}{2+\beta_{i, j}} \\
& \times\left[\frac{F_{i+1, j+1}^{x}-F_{i+1, j}^{x}}{\widehat{h}_{j}}-\left(\frac{F_{i+1, j}^{x y}}{2+\widehat{\alpha}_{i, j}}+\frac{F_{i+1, j+1}^{x y}}{2+\widehat{\beta}_{i, j}}\right)\right], \\
C_{2,3}= & \widehat{\Delta}_{i+1, j}-\left(\frac{F_{i+1, j}^{y}}{2+\widehat{\alpha}_{i, j}}+\frac{F_{i+1, j+1}^{y}}{2+\widehat{\beta}_{i, j}}\right) .
\end{aligned}
$$

From (43) and (50), we can see that a sufficient condition for $R_{x}(x, y)>0$ and $R_{y}(x, y)>0$ is as follows:

$$
C_{l, k}>0, \quad \widehat{C}_{l, k}>0, \quad l=0,1,2 ; k=0,1,2,3 .
$$

For $C_{0, k}>0, k=0,1,2,3$, a sufficient condition is

$$
\begin{aligned}
& \alpha_{i, j}>\max \left\{0,-2-\frac{h_{i} F_{i, j}^{x y}}{F_{i, j}^{y}}\right\}, \\
& \beta_{i, j}>\max \left\{0,-2+\frac{h_{i} F_{i+1, j}^{x y}}{F_{i+1, j}^{y}}\right\} .
\end{aligned}
$$

For $C_{1, k}>0, k=0,1,2,3$, a sufficient condition is

$$
\begin{aligned}
& \alpha_{i, j}>\max \left\{0,-2-\frac{h_{i} F_{i, j+1}^{x y}}{F_{i, j+1}^{y}}\right\}, \\
& \beta_{i, j}>\max \left\{0,-2+\frac{h_{i} F_{i+1, j+1}^{x y}}{F_{i+1, j+1}^{y}}\right\} .
\end{aligned}
$$

In order to give a simple sufficient condition for $C_{2, k}>0$, $k=0,1,2,3$, we rewrite the expressions of $C_{2, k}>0, k=$ $0,1,2,3$, as the following forms:

$$
\begin{aligned}
& C_{2,0}=\left(\frac{\widehat{\Delta}_{i, j}}{2}-\frac{F_{i, j}^{y}}{2+\widehat{\alpha}_{i, j}}\right)+\left(\frac{\widehat{\Delta}_{i, j}}{2}-\frac{F_{i, j+1}^{y}}{2+\widehat{\beta}_{i, j}}\right), \\
& C_{2,1}=\left(\frac{\widehat{\Delta}_{i, j}}{4}-\frac{F_{i, j}^{y}}{2+\widehat{\alpha}_{i, j}}\right)+\left(\frac{\widehat{\Delta}_{i, j}}{4}-\frac{F_{i, j+1}^{y}}{2+\widehat{\beta}_{i, j}}\right) \\
& +\left[\frac{\widehat{\Delta}_{i, j}}{4}+\frac{h_{i}\left(F_{i, j+1}^{x}-F_{i, j}^{x}\right)}{\hat{h}_{j}\left(2+\alpha_{i, j}\right)}\right] \\
& +\left[\frac{\widehat{\Delta}_{i, j}}{8}-\frac{h_{i} F_{i, j}^{x y}}{\left(2+\alpha_{i, j}\right)\left(2+\widehat{\alpha}_{i, j}\right)}\right] \\
& +\left[\frac{\widehat{\Delta}_{i, j}}{8}-\frac{h_{i} F_{i, j+1}^{x y}}{\left(2+\alpha_{i, j}\right)\left(2+\widehat{\beta}_{i, j}\right)}\right] \text {, } \\
& C_{2,2}=\left(\frac{\widehat{\Delta}_{i+1, j}}{4}-\frac{F_{i+1, j}^{y}}{2+\widehat{\alpha}_{i, j}}\right)+\left(\frac{\widehat{\Delta}_{i+1, j}}{4}-\frac{F_{i+1, j+1}^{y}}{2+\widehat{\beta}_{i, j}}\right) \\
& +\left[\frac{\widehat{\Delta}_{i+1, j}}{4}-\frac{h_{i}\left(F_{i+1, j+1}^{x}-F_{i+1, j}^{x}\right)}{\widehat{h}_{j}\left(2+\beta_{i, j}\right)}\right] \\
& +\left[\frac{\widehat{\Delta}_{i+1, j}}{8}+\frac{h_{i} F_{i+1, j}^{x y}}{\left(2+\beta_{i, j}\right)\left(2+\widehat{\alpha}_{i, j}\right)}\right] \\
& +\left[\frac{\widehat{\Delta}_{i+1, j}}{8}+\frac{h_{i} F_{i+1, j+1}^{x y}}{\left(2+\beta_{i, j}\right)\left(2+\widehat{\beta}_{i, j}\right)}\right] \text {, } \\
& C_{2,3}=\left(\frac{\widehat{\Delta}_{i+1, j}}{2}-\frac{F_{i+1, j}^{y}}{2+\widehat{\alpha}_{i, j}}\right)+\left(\frac{\widehat{\Delta}_{i+1, j}}{2}-\frac{F_{i+1, j+1}^{y}}{2+\widehat{\beta}_{i, j}}\right) \text {. }
\end{aligned}
$$


Thus, we can easily obtain the following sufficient condition for $C_{2, k}>0, k=0,1,2,3$ :

$$
\begin{gathered}
\alpha_{i, j}>\max \left\{0,-2-\frac{4 h_{i}\left(F_{i, j+1}^{x}-F_{i, j}^{x}\right)}{\left.\widehat{h}_{j} \widehat{\Delta}_{i, j}\right\},},\right. \\
\beta_{i, j}>\max \left\{0,-2+\frac{h_{i}\left(F_{i+1, j+1}^{x}-F_{i+1, j}^{x}\right)}{\widehat{h}_{j} \widehat{\Delta}_{i+1, j}}\right\}, \\
\widehat{\alpha}_{i, j}>\max \left\{0,-2+\frac{4 F_{i, j}^{y}}{\widehat{\Delta}_{i, j}},-2+\frac{4 F_{i+1, j}^{y}}{\widehat{\Delta}_{i+1, j}},\right. \\
\left.-2+\frac{4 h_{i} F_{i, j}^{x y}}{\widehat{\Delta}_{i, j}},-2-\frac{4 h_{i} F_{i+1, j}^{x y}}{\widehat{\Delta}_{i+1, j}}\right\}, \\
\widehat{\beta}_{i, j}>\max \left\{\begin{array}{l}
0,-2+\frac{4 F_{i, j+1}^{y}}{\widehat{\Delta}_{i, j}},-2+\frac{4 F_{i+1, j+1}^{y}}{\widehat{\Delta}_{i+1, j}}, \\
\left.-2+\frac{4 h_{i} F_{i, j+1}^{x y}}{\widehat{\Delta}_{i, j}},-2-\frac{4 h_{i} F_{i+1, j+1}^{x y}}{\widehat{\Delta}_{i+1, j}}\right\} .
\end{array}\right.
\end{gathered}
$$

By comparing the expressions of $\widehat{C}_{l, k}$ with those of $C_{l, k}$, we can easily obtain sufficient conditions for $\widehat{C}_{l, k}>0$ analogous to those for $C_{l, k}>0$. We summarize the above discussion as the following theorem.

Theorem 3. The biquartic rational interpolation spline defined in (24) is monotone if the shape parameters $\alpha_{i, j}, \beta_{i, j}, \widehat{\alpha}_{i, j}$, and $\widehat{\beta}_{i, j}$ satisfy the following conditions:

$$
\begin{aligned}
& \alpha_{i, j}=p_{i, j} \\
& +\max \left\{0,-2-\frac{h_{i} F_{i, j}^{x y}}{F_{i, j}^{y}},-2-\frac{h_{i} F_{i, j+1}^{x y}}{F_{i, j+1}^{y}},\right. \\
& -2+\frac{4 F_{i, j}^{x}}{\Delta_{i, j}},-2+\frac{4 F_{i, j+1}^{x}}{\Delta_{i, j+1}},-2+\frac{4 \widehat{h}_{j} F_{i, j}^{x y}}{\Delta_{i, j}}, \\
& \left.-2-\frac{4 \widehat{h}_{j} F_{i, j+1}^{x y}}{\Delta_{i, j+1}},-2-\frac{4 h_{i}\left(F_{i, j+1}^{x}-F_{i, j}^{x}\right)}{\widehat{h}_{j} \widehat{\Delta}_{i, j}}\right\} \text {, }
\end{aligned}
$$

$\beta_{i, j}=q_{i, j}$

$$
\begin{aligned}
+\max \{ & 0,-2+\frac{h_{i} F_{i+1, j}^{x y}}{F_{i+1, j}^{y}},-2+\frac{h_{i} F_{i+1, j+1}^{x y}}{F_{i+1, j+1}^{y}}, \\
& -2+\frac{4 F_{i+1, j}^{x}}{\Delta_{i, j}},-2+\frac{4 F_{i+1, j+1}^{x}}{\Delta_{i, j+1}}, \\
& -2+\frac{4 \widehat{h}_{j} F_{i+1, j}^{x y}}{\Delta_{i, j}},-2-\frac{4 h_{j} F_{i+1, j+1}^{x y}}{\Delta_{i, j+1}}, \\
& \left.-2+\frac{4 h_{i}\left(F_{i+1, j+1}^{x}-F_{i+1, j}^{x}\right)}{\widehat{h}_{j} \widehat{\Delta}_{i+1, j}}\right\},
\end{aligned}
$$

$$
\begin{aligned}
& \widehat{\alpha}_{i, j}=\widehat{p}_{i, j} \\
& +\max \left\{0,-2-\frac{\widehat{h}_{j} F_{i, j}^{x y}}{F_{i, j}^{x}},-2-\frac{\widehat{h}_{j} F_{i+1, j}^{x y}}{F_{i+1, j}^{x}},\right. \\
& -2+\frac{4 F_{i, j}^{y}}{\widehat{\Delta}_{i, j}},-2+\frac{4 F_{i+1, j}^{y}}{\widehat{\Delta}_{i+1, j}},-2+\frac{4 h_{i} F_{i, j}^{x y}}{\widehat{\Delta}_{i, j}}, \\
& \left.-2-\frac{4 h_{i} F_{i+1, j}^{x y}}{\widehat{\Delta}_{i+1, j}},-2-\frac{4 \widehat{h}_{j}\left(F_{i+1, j}^{y}-F_{i, j}^{y}\right)}{h_{i} \Delta_{i, j}}\right\}, \\
& \widehat{\beta}_{i, j}=\widehat{q}_{i, j} \\
& +\max \left\{0,-2+\frac{\widehat{h}_{j} F_{i, j+1}^{x y}}{F_{i, j+1}^{x}},-2+\frac{\widehat{h}_{j} F_{i+1, j+1}^{x y}}{F_{i+1, j+1}^{x}},\right. \\
& -2+\frac{4 F_{i, j+1}^{y}}{\widehat{\Delta}_{i, j}},-2+\frac{4 F_{i+1, j+1}^{y}}{\widehat{\Delta}_{i+1, j}}, \\
& -2+\frac{4 h_{i} F_{i, j+1}^{x y}}{\widehat{\Delta}_{i, j}},-2-\frac{4 h_{i} F_{i+1, j+1}^{x y}}{\widehat{\Delta}_{i+1, j}}, \\
& \left.-2+\frac{4 \widehat{h}_{j}\left(F_{i+1, j+1}^{y}-F_{i, j+1}^{y}\right)}{h_{i} \Delta_{i, j+1}}\right\} \text {, }
\end{aligned}
$$

where $p_{i, j}, q_{i, j}, \widehat{p}_{i, j}, \widehat{q}_{i, j}>0$ are free parameters for interactively adjusting the shape of the monotonicity preserving interpolation spline surface.

\section{Numeric Experiments}

In this section, we will construct a positivity preserving surface and two monotonicity preserving surfaces from the corresponding data with the biquartic rational interpolation spline and show the surfaces generated by the methods in $[5,6]$ for comparisons. Our method provides a set of simpler basis functions to represent the positive or monotonic surface data than $[5,6]$. In particular, it is worth noting that only four parameters are used in our method, that is, half of those used in $[5,6]$.

The positive surface data set in Table 1 is obtained from [5]. In Figure 1, the first three rows show the biquartic rational interpolation spline with different shape parameters for the positive surface data set given in Table 1, and the last row shows the surface constructed by the method in [5]. The images on the right column present the $x z$-view of the left. From the results, it can be seen that the biquartic interpolation spline describes the positive surface data set as well as [5].

The monotonic surface data sets in Table 2 are derived from [6]. In Figure 2, for visualizing the monotonic surface data set given in Table 2, the first three rows are the biquartic interpolation spline surfaces with different shape parameters, and the last row is the spline surface in [6]. The right column images are the $y z$-views of the left images. From the figure, it can be seen that the biquartic interpolation spline visualizes the monotonic surface data set as well as [6]. 

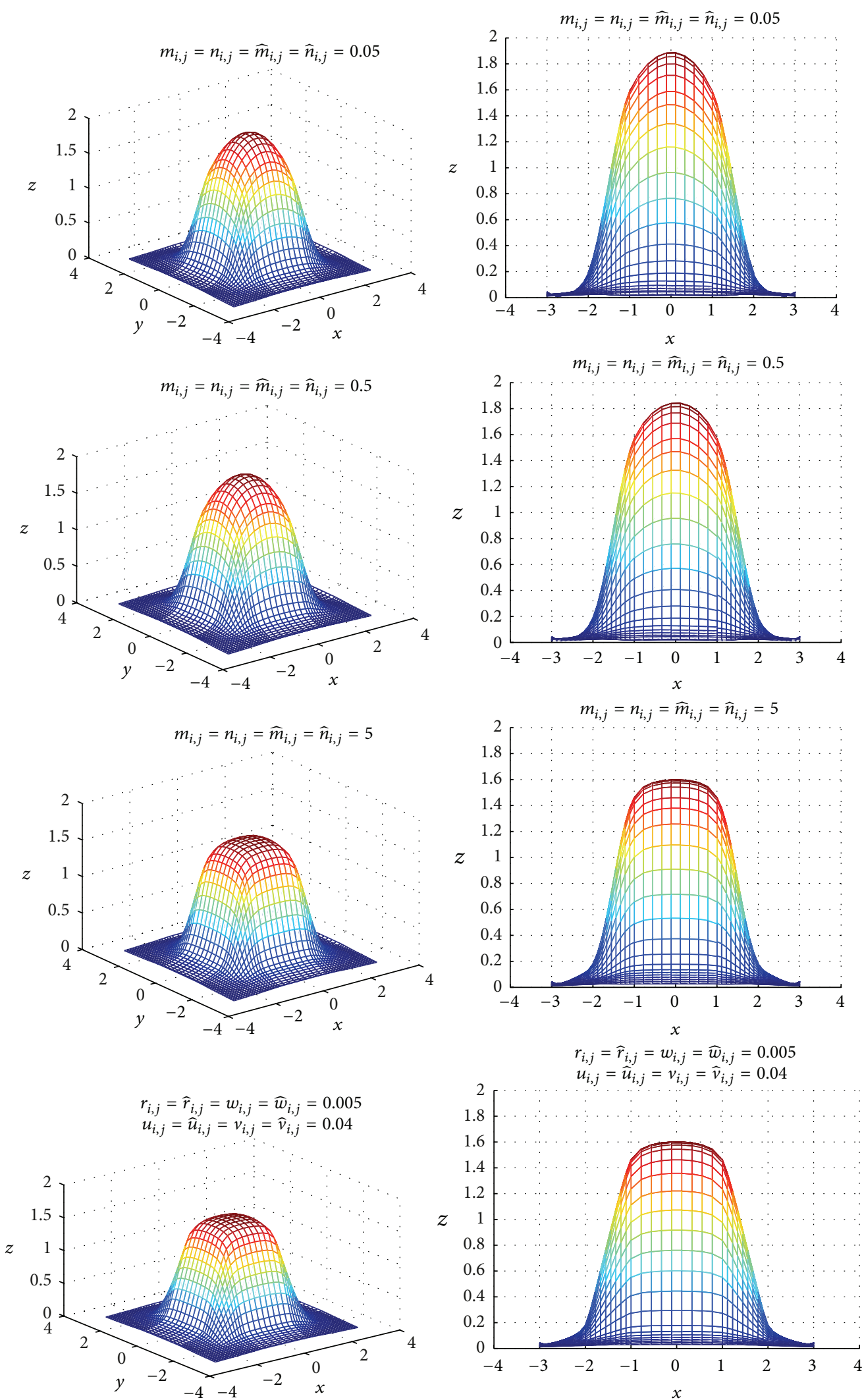

FIGURE 1: Surfaces generated from the given positive surface data set in Table 1. The first three rows are our biquartic rational interpolation spline surfaces with different shape parameters, and the last row is the surface in [5] with given parameters. 

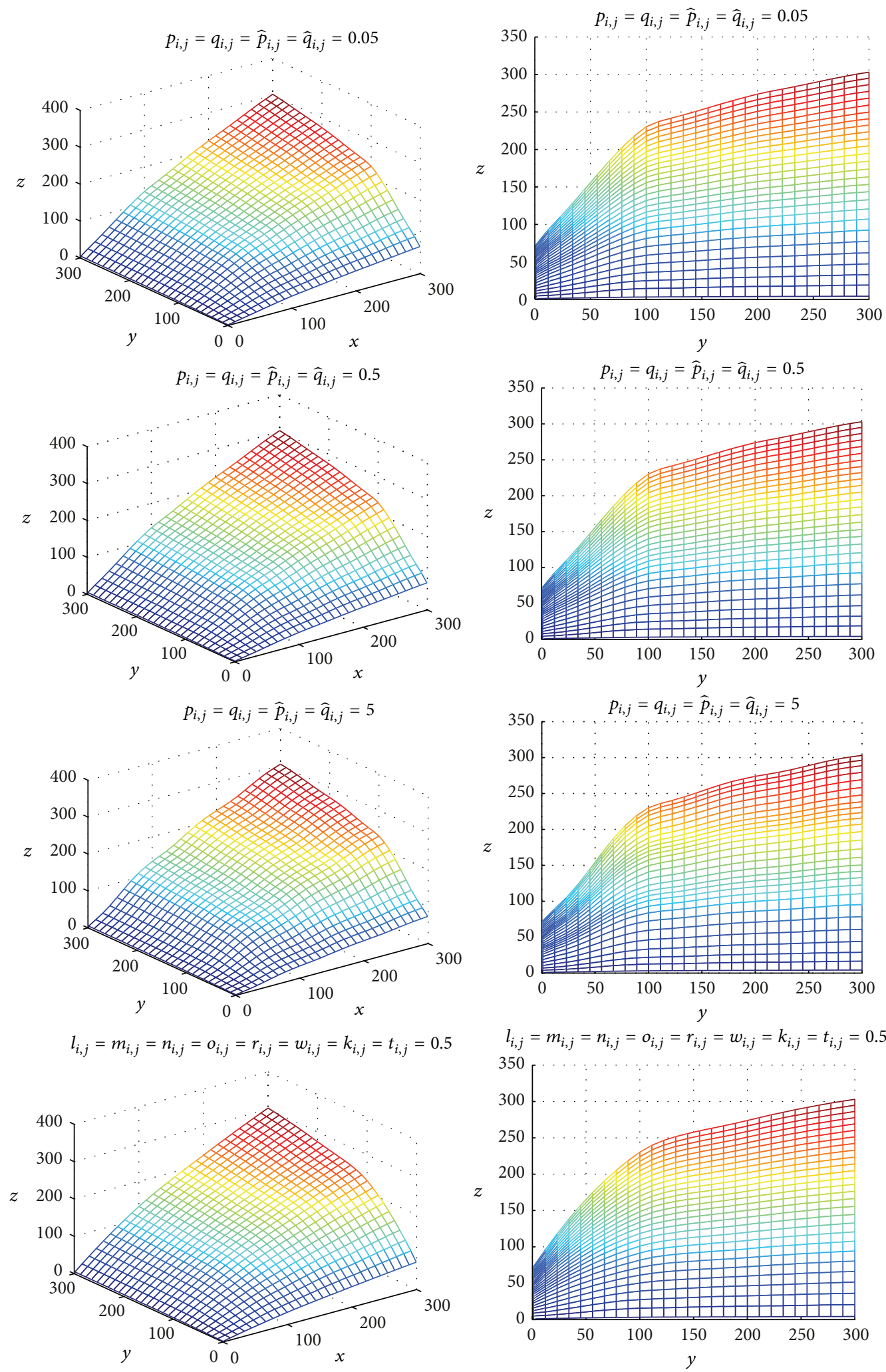

FIGURE 2: Surfaces created from the given monotonic surface data set in Table 2. The first three rows are our biquartic rational interpolation spline surfaces with different shape parameters, and the last row is the surface in [6] with given parameters. 
TABLE 1: Positive surface data set.

\begin{tabular}{lcccccc}
\hline$y / x$ & -3 & -2 & -1 & 1 & 2 & 3 \\
\hline-3 & 0.0124 & 0.0238 & 0.0404 & 0.0404 & 0.0238 & 0.0124 \\
-2 & 0.0238 & 0.0635 & 0.1667 & 0.1667 & 0.0635 & 0.0238 \\
-1 & 0.0404 & 0.1667 & 1.3333 & 1.3333 & 0.1667 & 0.0404 \\
1 & 0.0404 & 0.1667 & 1.333 & 1.3333 & 0.1667 & 0.0404 \\
2 & 0.0238 & 0.0635 & 0.1667 & 0.1667 & 0.0635 & 0.0238 \\
3 & 0.0124 & 0.0238 & 0.0404 & 0.0404 & 0.0238 & 0.0124 \\
\hline
\end{tabular}

TABLE 2: Monotonic surface data set.

\begin{tabular}{lcccc}
\hline$y / x$ & 1 & 100 & 200 & 300 \\
\hline 1 & 1.0100 & 31.9390 & 53.7148 & 72.8052 \\
100 & 3.1939 & 101.0000 & 169.8611 & 230.2302 \\
200 & 3.7982 & 120.1099 & 202.0000 & 273.7914 \\
300 & 4.2034 & 132.9235 & 223.5497 & 303.0000 \\
\hline
\end{tabular}

\section{Conclusion}

As stated above, the biquartic rational spline surface over rectangular domain includes the classical bicubic Coons surface as a special case. By using the quartic rational basis function, it can be rewritten neatly. Moreover, by selecting suitable parameters on the spline, it can be used to nicely visualize positive or monotonic surface data. Moreover, in our future works, we will concentrate on trying to make use of the modeling technique in practical applications, such as shape preserving surface reconstruction from 3D scattered data.

\section{Conflict of Interests}

The authors declare that there is no conflict of interests regarding the publication of this paper.

\section{Acknowledgments}

The research is supported by the National Natural Science Foundation of China (nos. 61173119 and 11271376), the Open Project Program of the State Key Lab of CAD\&CG (no. A1414) at Zhejiang University, Program for New Century Excellent Talents in University (no. NCET-13-0590), Graduate Students Scientific Research Innovation Project of Hunan Province (no. CX2012B111), and Mathematics and Interdisciplinary Sciences Project, Central South University. Science and technology project of Hunan Province (no. 2014FJ2008).

\section{References}

[1] R. K. Beatson and Z. Ziegler, "Monotonicity preserving surface interpolation," SIAM Journal on Numerical Analysis, vol. 22, no. 2, pp. 401-411, 1985.

[2] P. Costantini and F. Fontanella, "Shape-preserving bivariate interpolation," SIAM Journal on Numerical Analysis, vol. 27, no. 2, pp. 488-506, 1990.

[3] P. Costantini and C. Manni, "A bicubic shape-preserving blending scheme," Computer Aided Geometric Design, vol. 13, no. 4, pp. 307-331, 1996.
[4] L. U. Han and L. L. Schumaker, "Fitting monotone surfaces to scattered data using $C^{1}$ piecewise cubics," SIAM Journal on Numerical Analysis, vol. 34, no. 2, pp. 569-585, 1997.

[5] M. Z. Hussain and M. Sarfraz, "Positivity-preserving interpolation of positive data by rational cubics," Journal of Computational and Applied Mathematics, vol. 218, no. 2, pp. 446-458, 2008.

[6] M.Z. Hussain and M. Hussain, "Visualization of data preserving monotonicity," Applied Mathematics and Computation, vol. 190, no. 2, pp. 1353-1364, 2007.

[7] F. Ibraheem, M. Hussain, M. Z. Hussain, and A. A. Bhatti, "Positive data visualization using trigonometric function," Journal of Applied Mathematics, vol. 2012, Article ID 247120, 19 pages, 2012.

[8] X. Peng, Z. Li, and Q. Sun, "Nonnegativity preserving interpolation by $C^{1}$ bivariate rational spline surface," Journal of Applied Mathematics, vol. 2012, Article ID 624978, 11 pages, 2012.

[9] Q. Duan, H. Zhang, Y. Zhang, and E. H. Twizell, "Error estimation of a kind of rational spline," Journal of Computational and Applied Mathematics, vol. 200, no. 1, pp. 1-11, 2007. 


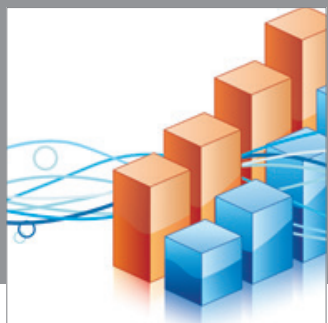

Advances in

Operations Research

mansans

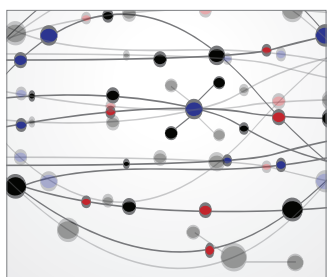

The Scientific World Journal
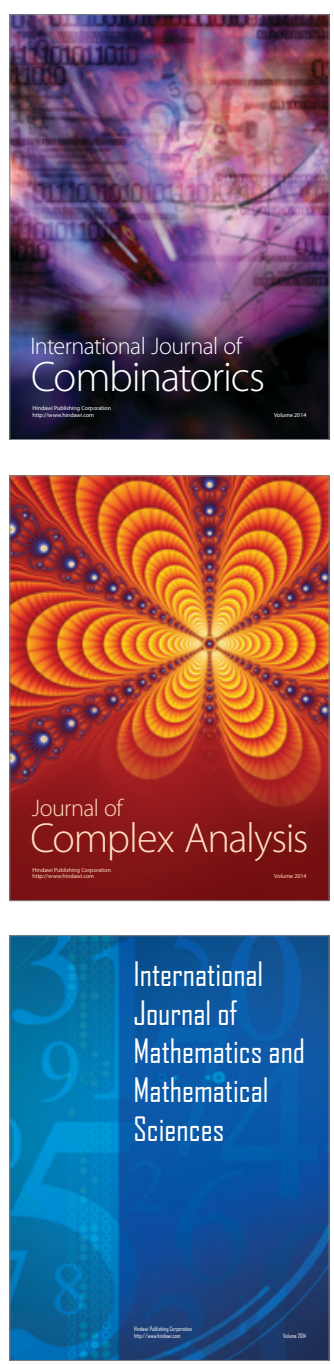
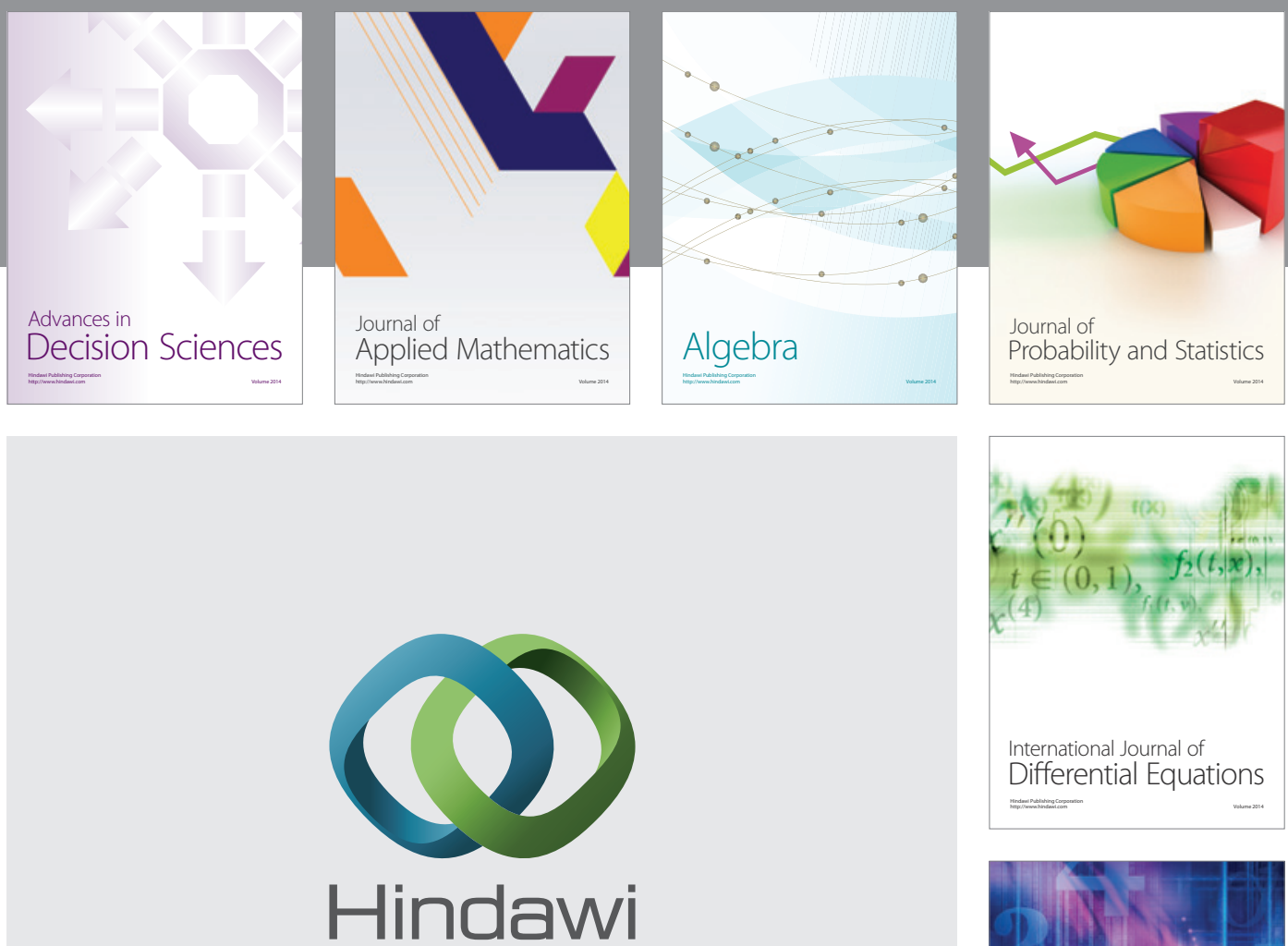

Submit your manuscripts at http://www.hindawi.com
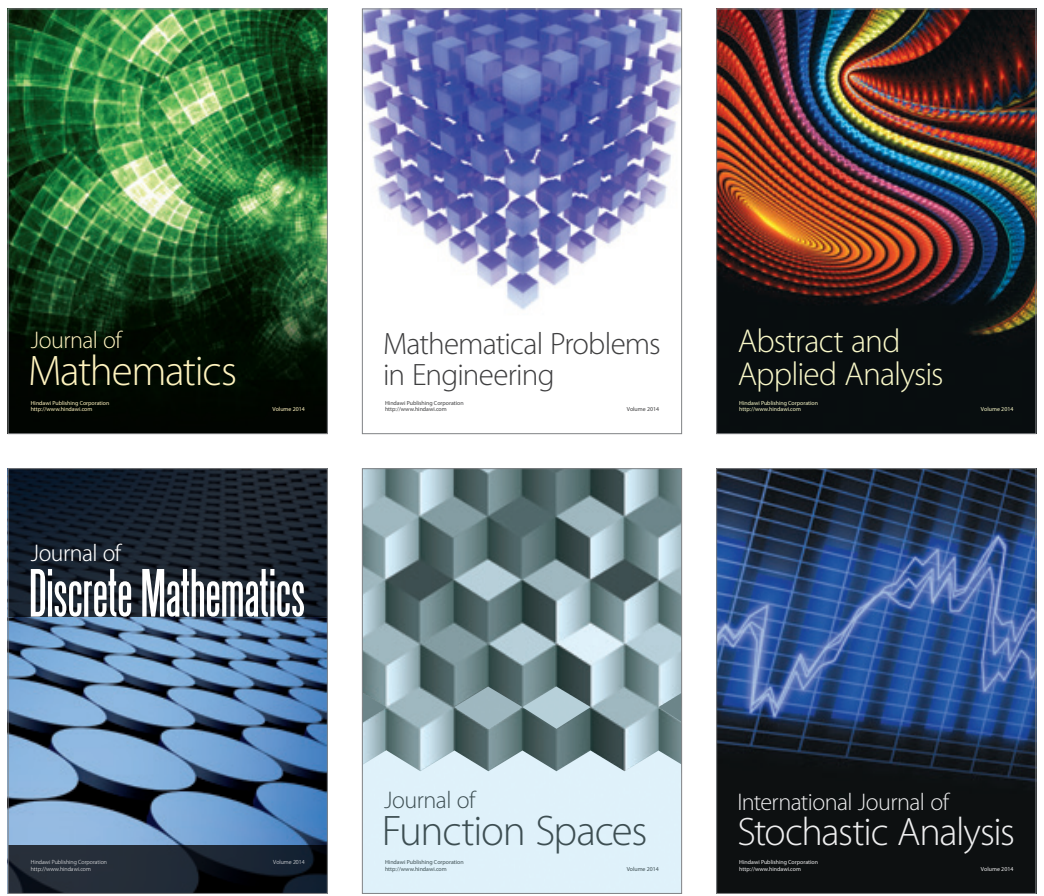

Journal of

Function Spaces

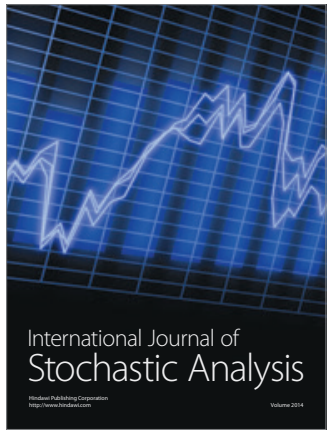

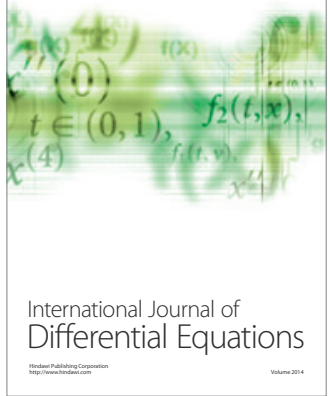
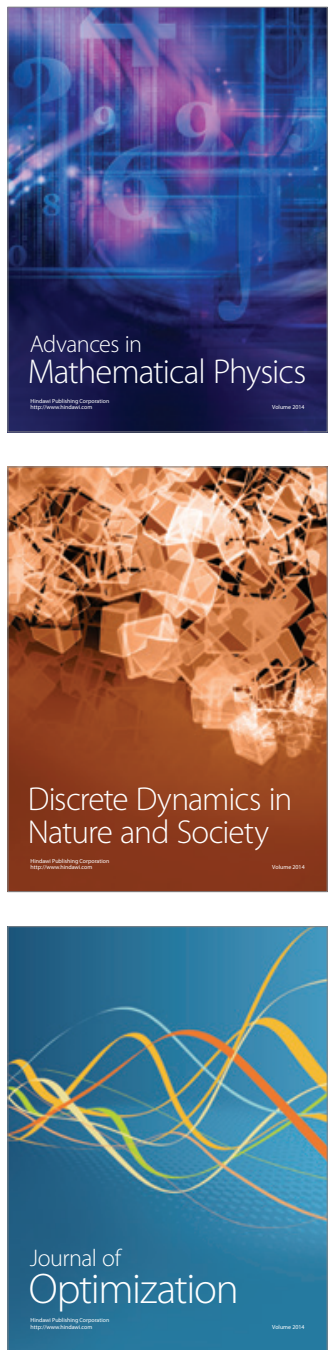